\title{
NON-DESTRUCTIVE RADIATION TESTING OF PHYSICAL AND MECHANICAL PROPERTIES OF SOLIDS ${ }^{\#}$
}

\author{
V.Deruyga, A. Kalinichenko, Yu. Kresnin, G.Popov*, \\ Kharkiv State University, Kharkiv, Ukraine
}

\begin{abstract}
The radiation-acoustic analyzer for real-time nondestructive investigation of phase state, mechanical and thermodynamic characteristics of solids was designed. Its action is based on original radiation-acoustic method, which includes probing of investigated materials by pulsed electron beam and registration of the excited acoustic waves. The results of radiation testing of some metallic alloys are discussed.
\end{abstract}

\section{INTRODUCTION}

The radiation-acoustic analyzer (RAA) has been developed for real-time simultaneous measurements of the Gruneisen parameter, sound velocity, elasticity modulus, the Poisson ratio, linear thermal expansion coefficient and specific heat of structural materials exposed to dynamic and static stresses, radiation and electromagnetic fields, temperature etc. It also may be used for investigation of kinetics of structure phase transition (SPT) of the first and second kind in metals, ceramics or polymers. Developed facility is especially useful for investigation of materials showing nonlinear behaviour in the vicinity of SPT where conventional static methods may not reveal some nonlinear effects.

\section{RADIATION-ACOUSTIC METHOD OF MATERIAL INVESTIGATION}

Radiation-acoustic method of measurement of characteristics of materials [1-7] includes the probing of investigated material by pulsed beam of ionizing particles (electrons, protons, gamma-quanta etc.) and registration of instantaneous acoustic stresses $\sigma(\boldsymbol{x}-\boldsymbol{s t})$ caused by fast heating of the material. Here $\boldsymbol{x}$ - distance between the center of the beam-target interaction spot and the acoustic detector, $\boldsymbol{s}$ - sound velocity, $\boldsymbol{t}$ - time. The magnitude of $\sigma$ is determined by the density $\mathcal{E}$ of energy, released by the beam in the target, the Gruneisen parameter $\Gamma$ and the Poisson ratio $\mu$ of the target material:

$$
\sigma(x-s t)=\gamma \varepsilon(x-s t) / 2,
$$

where $\gamma$ is the sound generation parameter, which is expressed through $\Gamma$ and $\mu$ as [2,3]:

\footnotetext{
${ }^{\#}$ Work was supported by the STCU, Kyiv, Grant \#155

*Email: popov@pht.univer.kharkov.ua
}

$$
\gamma_{1}=(1-2 \mu) \Gamma
$$

for one-dimensional (thin rod shaped) target,

$$
\gamma_{2}=\Gamma(1-2 \mu) /(1-\mu)
$$

for two-dimensional (thin plate shaped) target,

$$
\gamma_{3}=\Gamma
$$

for three-dimensional target (in bulk of the material). The Gruneisen parameter is given by the formula

$$
\Gamma=\alpha \mathbf{E}_{\mathrm{Y}} /[3 \rho \mathbf{C}(1-2 \mu)],
$$

where $\alpha$ is the coefficient of thermal expansion, $\mathbf{E}_{\mathrm{Y}}$ is the Young modulus, $\rho$ is the target material density, $\mathbf{C}$ is its specific heat.

By processing of the signal $\sigma(x-s t)$ registered by acoustic detector connected to the target one can extract information about those characteristics of the target material which determine the stress magnitude. The time resolution of the method is determined by the particle pulse width, beam diameter, geometry and the bandwidth of the detector. For our equipment it is limited by $10 \mathrm{~ns}$. Equation (1) is valid in the approximation of instant release of the energy in the target, i.e. $\tau \ll \tau_{s}=\mathbf{D} / \boldsymbol{s}$, where $\tau_{s}$ is the acoustic relaxation time, $\tau$ is the duration of the radiation pulse, and $\mathbf{D}$ is the beam diameter. It is also supposed that the condition

$$
\gamma(\mathbf{T}+\Delta \mathbf{T})-\gamma(\mathbf{T})<1
$$

is satisfies. It means that in the range of radiation overheating $\Delta \mathbf{T}=\varepsilon /(\rho \mathbf{C})$ parameter $\gamma$, does not change significantly. $\mathbf{T}$ is the initial temperature of the material.

\section{RADIATION-ACOUSTIC ANALYZER}

The radiation-acoustic analyzer includes an electron accelerator, a testing bench on which the tested sample is exposed to programmed sequence of external actions and the radiation-acoustic system (See Fig.1).

The radiation-acoustic system includes a set of acoustic sensors and the sensors of temperature, static and dynamic 
stress, deformation, radiation flux, etc. The data acquisition and processing module of the system is controlled by IBM PC computer and consists of a strobig analog-digital convertor, a commutator of analog signals, a module of synchronization and automated remote control.

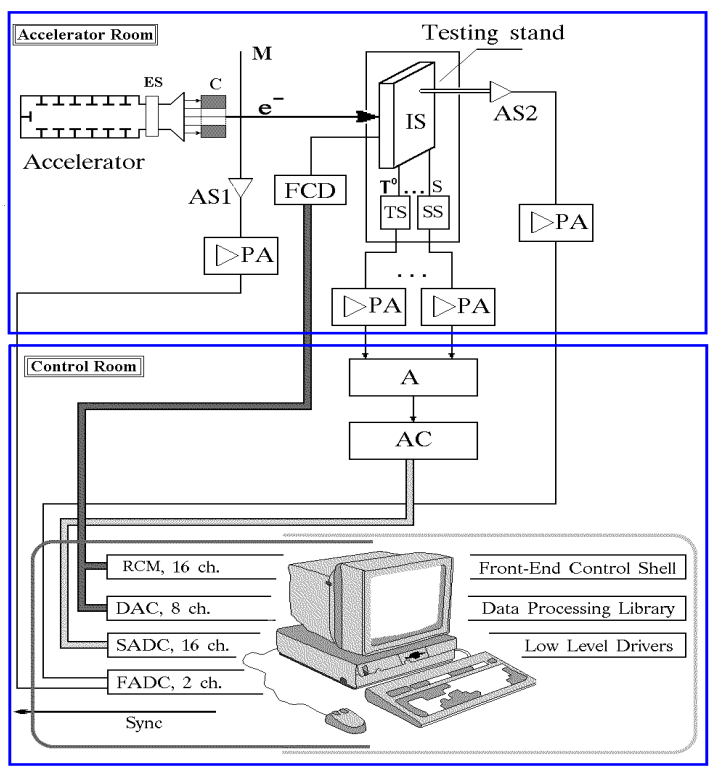

Figure 1. Scheme of the radiation-acoustic analyzer. $\mathrm{e}^{-}$- pulsed electron beam; ES - electromagnetic scanner; C -collimator; M - thermoacoustic monitor; AS1, AS2 acoustic sensors; IS - investigated sample; TS temperature sensor; SS - sensor of static and dynamic stresses; PA - preamplifier; FCD - set of final control devises; A - amplifiers; AC - commutator; SADC - high precision analog-digital converter; FADC - strobing analog-digital converter; Sync - clock pulse; DAC digital-analog converter; RCM - remote control module.

Radiation probing of investigated samples is performed by single pulses of electron beam with energy 5-30 MeV and low-intensity $\left(10^{7}-10^{10}\right.$ electrons in a pulse). The beam diameter is $1-4 \mathrm{~cm}$, the pulse width $10^{-8}-10^{-5} \mathrm{~s}$. Radiation heating $\Delta \mathrm{T} \leq 10^{-3} \mathrm{~K}$ of the sample material by such a pulse does not cause irreversible changes of investigated materials. To simplify the data analysis experimental samples are shaped as thin rods or plates. The sample thickness $\mathbf{d}$ must satisfy the conditions:

$$
\mathbf{d}\left(\mathrm{d} \mathbf{E}_{e} / \mathrm{d} \boldsymbol{x}\right)<\mathbf{E}_{e}, \quad \mathbf{d}+\boldsymbol{s} \tau_{b}<\mathbf{D}
$$

Here $\mathbf{E}_{e}$ is the electron energy, $\mathrm{d} \mathbf{E}_{e} / \mathrm{d} \boldsymbol{x}$ is the electron energy loss in the target material, $\tau_{b}$ is the effective electron pulse width, $\mathbf{D}$ - the beam diameter.

Acoustic pulses excited in the target IS and monitor $M$ by the same electron pulse are recorded using radiationresistant broad-band $(10 \mathrm{MHz})$ piezoceramic sensors AS1 and AS2, and analog-digital converter SADC (See Fig.1).
Amplitude, duration and shape of acoustic pulse and the delay between pulses from AS1 and AS2 are registered.

One- or two-dimensional thermoacoustic monitors $\mathrm{M}$ on the base of thin rods or plates allow as to determine the following characteristics of the beam: intensity, coordinates of the beam spot on the target and radial distribution of particles in the beam, instantaneous beam intensity, the energy of the beam pulse. The system also allows us to change programmatically the conditions of the experiment, for instance to change the temperature of the samples, static and dynamic stresses, radiation and electromagnetic field affecting the sample.

The software of the analyzer makes it possible to program the experimental routine, to control the experiment online, to read, process and display information. One can see on line the shape of acoustic signals, parameters of the acoustic pulse and the electron beam. Epures of temperature, stress and deformation can also be displayed on the monitor screen. The program calculates mechanical and thermodynamic characteristics of investigated materials, stores and reports the data.

\section{APPLICATION OF RAA FOR INVESTIGATION OF STRUCTURE PHASE TRANSITIONS IN SOLIDS}

The analyzer was used to study characteristics of materials with shape memory effect: $\mathrm{CuAlNi}$, TiNi, TiNiFe, TiNiCu, TiNiHf (the phase transition martensite $\leftrightarrow$ austenite); rare-earth metals: Gd, Dy (ferro $\leftrightarrow$ paramagnetic transition); hot-pressed high-temperature superconducting ceramics (the phase transition to a superconducting state); polymers: polymetylmetacrylate, polythetraftorethylene, polyethylene etc. (glassy $\leftrightarrow$ highly elastic state). Phase transitions were detected by the change of thermoelastic and thermodynamic parameters of the materials at the phase transition point. These studies were held in a wide range of temperature $(80-500 \mathrm{~K})$ and in a wide range of tensile or bending stress (10 - 1000 MPA).

The hysteresis behaviour of the temperature dependencies of parameters $\gamma(\mathbf{T}), \mathbf{E}_{\mathrm{Y}}(\mathrm{T}), \boldsymbol{s}(\mathbf{T}), \mathbf{C}(\mathbf{T})$ and $\alpha(\mathbf{T})$ was observed in the vicinity of the SPT for all investigated alloys with shape memory effect (CuAlNi, TiNi, TiNiFe, $\mathrm{TiNiCu}$, TiNiHf). Such hysteresis is characteristic of martensite austenite transitions. It is found that the shape of the hysteresis loop and its position on the temperature axis for parameters measured is depends on the concentration of the main components $\mathrm{Ti}, \mathrm{Ni}$ and the admixtures $\mathrm{Cu}$, Hf and $\mathrm{Fe}$. It also depends on the stress caused by the sample deformation. For TiNi based alloys the elasticity modulus $\mathbf{E}_{\mathrm{Y}}$ goes to a minimum in the region of SPT for both forward and backward transitions. It is connected with arising of "soft modes" in the vibration 
spectrum of the metal and ultimate instability of the crystal lattice near SPT.

On Fig.2 temperature dependencies of elasticity modulus $\mathbf{E}_{\mathrm{Y}}(\mathbf{T})$ for TiNiFe alloy in a free state (solid lines) and in a stress-strained state (dashed lines) are shown. External stress $\approx 120 \mathrm{MPa}$ was produced by a three-point bend at the temperature $280 \mathrm{~K}$. The stress raises the temperature of the martensitic phase transition by the value of $\Delta \mathbf{T} \leq 6 \mathrm{~K}$ and reduces a degree of "softening" of crystal lattice $\mathbf{E}_{\mathrm{m} 2}>\mathbf{E}_{\mathrm{m} 1}$. By integrating of specific heat $\mathbf{C}(\mathbf{T})$ in the vicinity of the phase transitions the heat of direct (exothermic) austenite-martensite SPT $\mathrm{H}_{\mathrm{A}-\mathrm{M}}=-18,3 \mathrm{~J} / \mathrm{g}$ and opposite (endothermic) martensite-austenite SPT $\mathrm{H}_{\mathrm{M}-\mathrm{A}}=16,9 \mathrm{~J} / \mathrm{g}$ were determined.



Figure 2. Temperature dependencies of the elasticity modulus $\mathbf{E}_{\mathrm{Y}}(\mathbf{T})$ for TiNiFe alloy.

Radiation-acoustic investigation of the alloys TiNi, $\mathrm{TiNiCu}$, TiNIFe at their electrochemical saturation with hydrogen $\mathrm{H}_{2}$ and deuterium $\mathrm{D}_{2}$ were carried out. Saturation of the alloy with hydrogen or deuterium changes the amplitude $\sigma$ of acoustic signal, and one can observe softening of the lattice and shifting of the hysteresis curves of $\gamma(\mathbf{T})$ and $\mathbf{E}_{\mathrm{Y}}(\mathbf{T})$ along the temperature axis by the value $\Delta \mathbf{T} \leq 30 \mathrm{~K}$ depending on concentration $\mathrm{H}_{2}$ or $\mathrm{D}_{2}$ concentration.

For hot pressed high-temperature superconducting ceramics the measurements of $\gamma(\mathbf{T})$ in the temperature range of 85-300 $\mathrm{K}$ were carried out. It was found that the maximum of the generation parameter $\gamma(\mathbf{T})$ is reached in the region of the superconducting phase transition $\left(\mathbf{T}_{\mathrm{k}}=92 \mathrm{~K}\right)$.

\section{CONCLUSION}

The major advantage of the radiation-acoustic method of the material properties investigation is its high productivity. Using this method one can obtain for an hour the amount of experimental data for obtaining of which by conventional methods one would have spend years. Therefore the method is extremely useful in technological the researches where the qualities of the end product depend on multiple parameters of the technological process. This method showed its power at the development of the technology of new polymer composite materials [8] where the amount of multiparameter experiments performed was so large, that it would have not be feasible without RAA.

\section{REFERENCES}

[1] White R.M., J. Appl. Phys. 34, 3559 - 3563 (1963).

[2] Zalubovskiy I.I., Kalinichenko A.I., Lazurik V.T. Introduction to radiation Acoustics (in Russian). Vishcha Shkola, Kharkov, 1986, p.86-111.

[3] Kalinichenko A.I., Popov G.F. Akusticheskiy Zhurnal. 36, 950-952 (1990).

[4] Popov G.F, Fizika Metallov i Metallovedenie. 75, 84-89 (1993).

[5] Kresnin Yu.A., Popov G.F. Instruments and Experimental

Techniques. 37, 592-595 (1994).

[6] Kresnin Yu.A., Popov G.F. Bulletin of the American Physical Society. 40, 1100 (1995).

[7]Popov G., Deryuga V., Kalinichenko A., Kresnin Yu. Proc. Intern. Conf. on Accelerator and Large Experimental Physics Control Systems. Chicago, USA, Oct.30, p.954-956, (1995).

[8] G. Popov, I. Zalubovskiy, A. Avilov, V. Rudychev, Bulletin of the American Physical Society 42, N3. p.1375, (1997). 